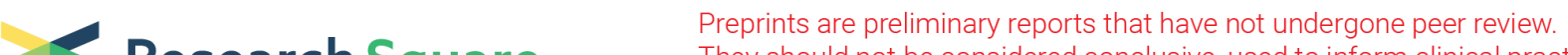 Research S Suare or referenced by the media as validated information. \\ Establishment and Evaluation of a Risk-prediction Model for HBP in Elderly Patients With NAFLD From a Health Management Perspective
}

\section{Xin Luo}

Department of health management, School of Public Health, Shanghai University of Traditional Chinese Medicine, Shanghai

\section{An Zhang}

Department of health management, School of Public Health, Shanghai University of Traditional Chinese Medicine, Shanghai

\section{Hong Pan}

Department of health management, School of Public Health, Shanghai University of Traditional Chinese Medicine, Shanghai

\section{Xinxin Shen}

Department of health management, School of Public Health, Shanghai University of Traditional Chinese Medicine, Shanghai

\section{Baocheng Liu}

Shanghai Collaborative Innovation Center of Traditional Chinese Medicine Health Service, Shanghai University of Traditional Chinese Medicine

\section{Dong Li}

Zhangjiang Community Health Service Centers, Pudong New Area, Shanghai

\section{Jijia Sun ( $\nabla_{\text {jijiasun@163.com ) }}$}

Shanghai Collaborative Innovation Center of Traditional Chinese Medicine Health Service, Shanghai University of Traditional Chinese Medicine

\section{Research Article}

Keywords: Nonalcoholic fatty liver, Hypertension, Lasso regression analysis, Random forest model, Risk factors, Nomogram, Health management

Posted Date: January 3rd, 2022

DOl: https://doi.org/10.21203/rs.3.rs-1188676/v1

License: (9) This work is licensed under a Creative Commons Attribution 4.0 International License. Read Full License 


\section{Abstract}

Objective: Elderly patients with nonalcoholic fatty liver disease (NAFLD) are at a higher risk of developing high blood pressure (HBP) and having a low quality of life. This study established an effective, individualised, early HBP risk-prediction model and proposed health management advice for the ${ }^{3} 60$ patients with NAFLD in Shanghai, China.

Methods: Questionnaire surveys, physical examinations, and biochemical tests were conducted on 7,319 cases of sample data. Risk factors were screened using the least absolute shrinkage and selection operator (Lasso) model and random forest (RF) model. A risk-prediction model was established using logistic regression analysis and dynamic nomogram was drawn. The model was evaluated for discrimination, calibration, and clinical applicability using receiver operating characteristic curves (ROC), calibration curves, decision curve analysis (DCA), net reclassification index (NRI), and external validation.

Results: The results suggested the model showed moderate predictive ability. The area under curve (AUC) of internal validation was 0.707 (95\% Cl: 0.688-0.727), the external validation AUC was $0.688(95 \% \mathrm{Cl}$ : $0.672-0.705)$. The calibration plots showed good calibration, the risk threshold of the decision curve was $30-56 \%$, and the NRI value was 0.109 .

Conclusion: This HBP risk factor model may be used in clinical practice to predict the HBP risk in NAFLD patients.

\section{Introduction}

Nonalcoholic fatty liver disease (NAFLD) is an acquired metabolic disease characterised by fatty deposits in the liver ${ }^{[1]}$. Its prevalence is increasing with the standard of living of the population ${ }^{[2]}$, and it is gradually becoming the most common chronic liver disease worldwide, posing an increasing economic and medical burden ${ }^{[3,4]}$. NAFLD prevalence is higher in China than in some developed countries because of the variability of disease prevalence and lifestyle changes. A projection of the future burden of NAFLD shows China is expected to have 314 million patients with NAFLD by $2030^{[5]}$.

High blood pressure (HBP) is a common cardiovascular disease with clinical diagnostic criteria of systolic blood pressure (SBP) $\geq 140 \mathrm{mmHg}$ or diastolic blood pressure (DBP) $\geq 90 \mathrm{~mm} \mathrm{Hg}{ }^{[6]}$, resulting in 9.4 million deaths worldwide ${ }^{[7]}$. China ranks first worldwide for deaths due to HBP, and the number of elderly people suffering from HBP is $>60 \%[8,9]$. Recent studies have found that the risk of hypertension is notably higher in patients with NAFLD than in other populations ${ }^{[10,11,12]}$, and there is a close relationship between the two. The prevalence of NAFLD combined with hypertension is approximately $39.34 \%[2,13,14]$, and the quality of life of patients with NAFLD-HBP is lower than either alone.

Used as a quantitative tool for assessing risks and benefits, the clinical prediction models (CPMs) can provide medical practitioners with intuitive and scientifically valid data; therefore, their application is 
becoming increasingly common ${ }^{[15]}$. However, most CPMs are established in developed countries, with only a few in developing and underdeveloped countries. Through a literature review, we found few reports on the establishment of the CPMs for HBP in China, and the CPMs in different regions are significantly different ${ }^{[7,16,17,18,19]}$. Therefore, it is of great practical value to establish CPMs for different regions and populations in China and propose health management strategies based on the research findings.

The HBP risk-prediction model for elderly patients with NAFLD developed in this study could, to some extent, fill the research gap in terms of region and population. Using multiple indicators obtained from questionnaires, physical examinations, and biochemical tests predict the risk of concurrent HBP in elderly patients with NAFLD and can help medical practitioners identify and select high-risk individuals for nonmedical health interventions at an early stage to delay or prevent NAFLD patients from developing HBP.

\section{Results}

\subsection{Basic statistical description}

The basic demographic information and clinical examination results of the study population were collected primarily through questionnaires, physical examinations, and biochemical tests. After processing, the final 24 basic variables in the analysis were: Age, SBP, DBP, body mass index (BMI), waistto-hip ratio (WHR), albumin (ALB), alanine aminotransferase (ALT), aspartate aminotransferase (AST), red blood cells (RBC), UREA, glucose (GLU), haemoglobin (HGB), platelets (PLT), total cholesterol (TC), total bilirubin (TB), creatinine (CRE), low-density lipoprotein (LDL) triglycerides (TG), uric acid (UA), alphafetoprotein (AFP), basophils (BASO), eosinophils (EOS), lymphocytes (LYMPH), and neutrophils (NEUT).

Among the samples collected in 2017, the data analyses of the 5,287 samples in this study are shown in Table 1, illustrating the basic statistical description of the sample data for NAFLD and non-NAFLD cases. The 24 variables in the analysis do not meet the normality by test due to the large sample size, mean \pm standard deviation is used for description, and Chi-square test and $t$-test were used to analyse whether the differences between groups were statistically significant. 
Table 1

Differences in demographic and clinical characteristics between the No-NAFLD and NAFLD groups. [mean $\pm \mathrm{SD}$ or $\mathrm{N}(\%)$ ]

\begin{tabular}{|c|c|c|c|c|}
\hline Demographic characteristics & $\begin{array}{l}\text { NAFLD } \\
(n=2845)\end{array}$ & $\begin{array}{l}\text { No-NAFLD } \\
(n=2442)\end{array}$ & $X^{2} / Z$ & $\mathbf{P}$ \\
\hline \multicolumn{5}{|l|}{ Hypertension } \\
\hline Yes & $1714(60.2 \%)$ & $1124(46.0 \%)$ & \multirow[t]{2}{*}{106.838} & \multirow[t]{2}{*}{$<0.001$} \\
\hline No & $1131(39.8 \%)$ & $1318(54.0 \%)$ & & \\
\hline Age (years) & $69.01 \pm 6.92$ & $69.39 \pm 7.36$ & 1.897 & $0.058^{*}$ \\
\hline BMI $\left(\mathrm{kg} / \mathrm{m}^{2}\right)$ & $25.37 \pm 2.87$ & $22.57 \pm 3.11$ & 33.730 & $<0.001$ \\
\hline $\mathrm{SBP}(\mathrm{mmHg})$ & $144.59 \pm 21.24$ & $139.33 \pm 22.80$ & 8.635 & $<0.001$ \\
\hline $\mathrm{DBP}(\mathrm{mmHg})$ & $82.26 \pm 11.35$ & $80.64 \pm 12.10$ & 5.017 & $<0.001$ \\
\hline WHR & $0.90 \pm 0.06$ & $0.86 \pm 0.06$ & 20.773 & $<0.001$ \\
\hline $\operatorname{ALB}(g / L)$ & $44.53 \pm 2.53$ & $43.96 \pm 2.71$ & 7.843 & $<0.001$ \\
\hline $\operatorname{ALT}(\mathrm{U} / \mathrm{L})$ & $25.97 \pm 13.94$ & $21.48 \pm 11.03$ & 13.071 & $<0.001$ \\
\hline AST $(U / L)$ & $23.74 \pm 8.06$ & $23.04 \pm 6.68$ & 3.467 & 0.001 \\
\hline $\operatorname{RBC}\left(10^{12} / \mathrm{L}\right)$ & $4.63 \pm 0.46$ & $4.51 \pm 0.48$ & 9.329 & $<0.001$ \\
\hline UREA (mmol/L) & $5.51 \pm 1.50$ & $5.43 \pm 1.53$ & 1.950 & $0.051^{*}$ \\
\hline GLU (mmol/L) & $6.38 \pm 1.80$ & $5.92 \pm 1.58$ & 9.826 & $<0.001$ \\
\hline HGB $(g / L)$ & $141.39 \pm 13.60$ & $138.68 \pm 14.44$ & 6.994 & $<0.001$ \\
\hline $\operatorname{PLT}\left(10^{9} / \mathrm{L}\right)$ & $200.69 \pm 54.66$ & $192.28 \pm 57.19$ & 5.438 & $<0.001$ \\
\hline $\mathrm{TC}(\mathrm{mmol} / \mathrm{L})$ & $5.11 \pm 0.96$ & $5.05 \pm 0.98$ & 2.111 & 0.035 \\
\hline TB $(\mu \mathrm{mol} / \mathrm{L})$ & $15.71 \pm 5.79$ & $15.81 \pm 5.62$ & 0.642 & 0.521 * \\
\hline CRE $(\mu \mathrm{mol} / \mathrm{L})$ & $68.76 \pm 17.74$ & $70.51 \pm 18.28$ & 3.526 & $<0.001$ \\
\hline LDL (mmol/L) & $3.21 \pm 0.86$ & $3.13 \pm 0.86$ & 3.634 & $<0.001$ \\
\hline TG (mmol/L) & $1.71 \pm 1.21$ & $1.21 \pm 9.75$ & 18.529 & $<0.001$ \\
\hline $\mathrm{UA}(\mu \mathrm{mol} / \mathrm{L})$ & $358.71 \pm 87.56$ & $327.51 \pm 82.15$ & 13.287 & $<0.001$ \\
\hline AFP (ng/mL) & $6.88 \pm 4.67$ & $6.89 \pm 5.60$ & 0.056 & $0.955^{\star}$ \\
\hline BASO $\left(10^{9} / \mathrm{L}\right)$ & $0.02 \pm 0.03$ & $0.03 \pm 0.05$ & 2.411 & 0.016 \\
\hline
\end{tabular}




\begin{tabular}{|lllll|}
\hline Demographic characteristics & $\begin{array}{l}\text { NAFLD } \\
(\mathbf{n}=\mathbf{2 8 4 5})\end{array}$ & $\begin{array}{l}\text { No-NAFLD } \\
(\mathbf{n}=\mathbf{2 4 4 2})\end{array}$ & $X^{2} / \mathbf{Z}$ & $\mathbf{P}$ \\
\hline EO $\left(10^{9} / \mathrm{L}\right)$ & $0.14 \pm 0.14$ & $0.13 \pm 0.12$ & 2.261 & 0.024 \\
\hline LYMPH $\left(10^{9} / \mathrm{L}\right)$ & $1.95 \pm 0.59$ & $1.79 \pm 1.31$ & 5.679 & $<0.001$ \\
\hline NEUT $\left(10^{9} / \mathrm{L}\right)$ & $3.40 \pm 1.14$ & $3.17 \pm 1.10$ & 7.378 & $<0.001$ \\
\hline note:"*" means $p>0.05$, and the difference was not statistically significant & \\
\hline
\end{tabular}

\subsection{Screening results of characteristic variables}

In the training set, nine characteristic variables out of 24 basic variables were screened using Lasso model and intersected with 18 characteristic variables screened using RF to obtain the common variables, age, SBP, BMI, ALT, UREA, TC, LDL, UA, and NEUT (Figures 2). Nine common variables were included in the multivariate logistic regression analysis, and the final characteristic variables were Age, SBP, BMI, ALT, UREA, UA, and NEUT after excluding those with $p>0.05$ (Figure 3, Table 2).

Table 2

Predictive factors for hypertension incidence risk in NAFLD patients

\begin{tabular}{|c|c|c|c|c|c|c|}
\hline \multirow{2}{*}{$\begin{array}{l}\text { Intercept } \\
\text { and variable }\end{array}$} & \multicolumn{6}{|c|}{ Prediction model } \\
\hline & $\beta$ & $Z$ value & P-value & OR & $2.5 \% \mathrm{Cl}$ & $97.5 \% \mathrm{Cl}$ \\
\hline Intercept & -9.534 & -14.86 & $<0.001$ & $7.237 e^{-05}$ & $2.029 e^{-05}$ & $2.512 e^{-04}$ \\
\hline Age & 0.024 & 3.79 & $<0.001$ & 1.024 & 1.012 & 1.037 \\
\hline SBP & 0.025 & 11.80 & $<0.001$ & 1.026 & 1.021 & 1.030 \\
\hline BMI & 0.109 & 7.12 & $<0.001$ & 1.116 & 1.083 & 1.150 \\
\hline ALT & 0.010 & 2.95 & 0.003 & 1.010 & 1.003 & 1.016 \\
\hline UREA & 0.077 & 2.63 & 0.008 & 1.080 & 1.020 & 1.143 \\
\hline UA & 0.002 & 4.78 & $<0.001$ & 1.002 & 1.001 & 1.003 \\
\hline NEUT & 0.120 & 3.20 & 0.001 & 1.127 & 1.048 & 1.214 \\
\hline
\end{tabular}

\subsection{Model construction and prediction results}

Using the seven indicators selected above as independent variables and "whether NAFLD patients have concurrent HBP" as dependent variables, a model was constructed using logistic regression to obtain a 
prediction model for the risk of concurrent HBP in elderly NAFLD patients:

$$
\begin{gathered}
y_{\text {model }}=-9.534+0.024 \cdot A g e+0.025 \cdot S B P+0.109 \cdot B M I+0.010 \cdot A L T \\
+0.077 \cdot U R E A+0.002 \cdot U A+0.120 \cdot N E U T
\end{gathered}
$$

HBP risk in patients with NAFLD can be simply and visually predicted based on the nomogram drawn from this model (Figure 4). For example, suppose a patient with NAFLD had the following characteristics:

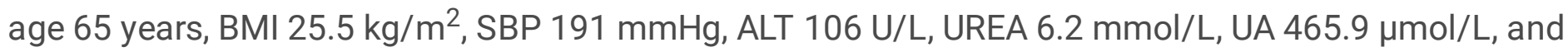
NEUT $4.2 \cdot 10^{9} / \mathrm{L}$, with a score of 322 , corresponding to a probability of 0.915 , indicating that the risk of concurrent HBP in this NAFLD patient is $91.5 \%$. In addition, we created an online app to predict the probability of concurrent HBP in elderly patients with NAFLD based on the model established in this study. The website is https://studentluo.shinyapps.io/DynNomapp/. This app can help clinicians diagnose HBP and community medical practitioners design reasonable and effective primary and secondary prevention management programs for NAFLD combined with HBP.

\subsection{Model evaluation and comparison}

To evaluate the discrimination, calibration, and clinical applicability of the risk nomogram established in this study, the model was validated. The AUC of internal validation set was 0.707 (95\% Cl: $0.688-0.727$ ) and the AUC of external validation set was 0.688 (95\% Cl: 0.672-0.705) (Figure 5). The calibration curves showed moderate agreement, and the results of the decision curves indicated that if the risk threshold of HBP in NAFLD patients was $30 \%-56 \%$, predicting the risk of HBP using this nomogram is more beneficial than implementing an intervention-all-patients scheme (Figure 6). In addition, we analysed each variable in the model and plotted the ROC curve; the results are shown in Figure 7, Table 3.

Table 3

The optimal cut-off value, specificity, and sensitivity of risk factors

\begin{tabular}{|lllll|}
\hline Characteristic & AUC & Cut-off value & Specificity (\%) & Sensitivity (\%) \\
\hline Age & 0.591 & 67.50 & 57.90 & 55.40 \\
SBP & 0.660 & 135.50 & 49.50 & 74.40 \\
BMI & 0.619 & 24.65 & 54.30 & 64.00 \\
ALT & 0.543 & 23.50 & 59.60 & 48.40 \\
UREA & 0.543 & 4.85 & 39.70 & 67.70 \\
UA & 0.580 & 360.15 & 63.00 & 49.10 \\
\hline NEUT & 0.558 & 3.05 & 47.60 & 61.20 \\
\hline
\end{tabular}


Employing the commonly used indicators, SBP and DBP, in HBP diagnosis, model I (AUC was 0.660, 95\% $\mathrm{Cl}: 0.636-0.685)$ containing only SBP and DBP was established in the NAFLD population. It was compared with the model constructed in this study using NRI, and the value was 0.109 , indicating that the model constructed in this study was better than model I and had better predictive ability (Figure 8).

\section{Discussion}

According to the prediction model, we established that seven indicators, Age, SBP, BMI, ALT, UREA, UA, and NEUT, are the risk factors for HBP development in patients with NAFLD.

Among previous studies on predictive models for the risk of developing HBP in China, a longitudinal study by Zhang et al. analysed 26,496 HBP patients and extracted 5 risk factors from 11 examined biomarkers to build a Hypertension Synthetic Predictor (HSP) for HBP, including Blood Pressure Factors (BPF) determined by SBP and DBP [29]. In a prospective study by Chen et al. that included 2,785 HBP patients, the variables in their established risk-prediction model were Age, BMI, SBP, DBP, and Fasting blood glucose (FBG) ${ }^{[30]}$. In addition, in a review study of HBP prediction models, 26 articles reporting on 48 prediction models were analysed (seven domestic studies; others from Europe, the United States, Korea, Japan, and Iran). The most common variables in the HBP prediction models included age, BMI, and blood pressure level, among others ${ }^{[16]}$. Most studies on HBP prediction models in China and abroad included the predictive variables of age, SBP, BMI (height, weight), etc., consistent with the results of this study.

Age is an irreversible risk factor for HBP development. Some studies have shown that HBP prevalence increases with an increase in age, and there is a strong correlation between biological ageing and elevated blood pressure ${ }^{[6,31,32,33]}$. SBP and DBP are diagnostic indicators commonly used for HBP. This study also found that SBP was one of the main predictors of concurrent HBP and NAFLD, and there is a correlation between $\mathrm{BMI}$ and $\mathrm{HBP}{ }^{[34,35]}$. The results of an $\mathrm{HBP}$ prediction model study based on 135,715 individuals showed that a lower BMI reduced the risk of developing $\mathrm{HBP}^{[17]}$. Among patients with $\mathrm{HBP}$ in the United States, $49.5 \%$ are obese $(\mathrm{BMI}>28)^{[36]}$. Moreover, there is strong evidence that obesity (including visceral obesity) is the most common risk factor for NAFLD ${ }^{[3,37]}$. The World Health Organization's BMI classification criteria are not suitable for application among Asians, and some studies have defined the BMI classification for a Chinese population as follows: underweight (<18.5), normal (18.5-22.9), overweight (23.0-27.5), and obese (>27.5) ${ }^{[38]}$. Based on such criteria, the obesity rate of subjects with NAFLD and HBP in the present study was $73.8 \%$.

Studies have shown that blood pressure is an important determinant of plasma UREA concentration. The higher the blood pressure, the higher the UREA concentration. In untreated HBP patients, elevated blood pressure may be caused by renal insufficiency ${ }^{[39]}$, and UREA abnormalities are also closely associated with kidney disease development ${ }^{[40,41]}$, suggesting that HBP is a cause or consequence of kidney disease $^{[42]}$. 
UA is associated with chronic kidney disease and cardiovascular disease and can be a predictor of developing HBP in adults ${ }^{[3,44]}$. It is positively correlated with the blood pressure level. An increase in UA implies an increased risk of future cardiovascular disease ${ }^{[21,45]}$. In a cross-sectional study of 33,570 patients with HBP by Ren et al., the established risk-prediction model included variables such as Gender, Age, Height, Weight, and UA ${ }^{[17]}$. Meanwhile, Rosa et al. proposed that UA is an important NAFLD predictor variable ${ }^{[46]}$.

The results of previous studies suggest that the development of cardiovascular diseases, including HBP, is closely associated with white blood cells (WBCs), a marker of inflammation $[45,47,48]$. An abnormal rise in WBC count may predict an increased risk of developing HBP, where NEUT is a major component of WBCs. A correlation between NEUT and HBP has also been noted in some studies, with higher NEUT values in patients with $\mathrm{HBP}^{[49]}$. In addition, some studies reported higher NEUT values in obese than in non-obese individuals ${ }^{[50,51]}$.

ALT is the main indicator abnormal liver function. It is present in the liver and is the main marker of liver injury ${ }^{[52]}$. The population in our study was mainly composed of patients with NAFLD, so the predictor ALT is included. A few studies have explored the association between ALT and HBP development, with equally few studies demonstrating association between ALT and HBP $[53,54,55]$. One of the cross-sectional studies conducted in Chinese adults suggested a positive linear correlation between ALT and HBP development.

Elevated blood pressure is strongly associated with increased dietary sodium intake ${ }^{[56,57,58]}$. In an article published in 2016, researchers conducted a dietary study across 20 Chinese provinces, which revealed that the per capita salt intake was $9.1 \mathrm{~g} /$ day and sodium intake was $5.4 \mathrm{~g} /$ day, both exceeding the recommended maximum daily salt intake $(6 \mathrm{~g} /$ day $)$ and sodium intake $(2 \mathrm{~g} / \text { day })^{[59]}$. Meanwhile, excessive intake of fats, sugars, and meat in the diet coupled with reduced physical activity are the main causes of increased BMI in the population ${ }^{[9]}$. A high-protein diet leads to excessive amino acids, which are oxidised into energy, in turn increasing UREA synthesis in the body ${ }^{[60]}$. To date, the basic treatment for excessive UA is dietary control, and a study by $R$ et al. suggested that increased intake of vitamins, such as vitamin $\mathrm{C}$ and $\mathrm{E}$, can reduce UA levels ${ }^{[43]}$. The Dietary Approaches to Stop Hypertension program (DASH), a healthy dietary model proposed by the United States in 1997, emphasises that adequate intake of vegetables, fruits, and low-fat (or skim) milk is essential, whereas excessive animal fats should be avoided. Some studies have confirmed the effectiveness of this approach ${ }^{[9,61,62]}$. Based on the results of the present study, a low sodium, low sugar, high vitamin $\mathrm{C}$ and $\mathrm{E}$, and moderate polyunsaturated fatty acid (PUFA) and protein diet should be selected for individuals with high HBP risk in patients with NAFLD, based on DASH, while emphasising an increase in moderate physical exercise.

To date, interventions such as dietary control, physical exercise, and weight loss for patients with NAFLD remain one of the main basic therapies to delay or prevent the progression of the disease ${ }^{[63,64]}$. In addition, lifestyle changes play an important role in controlling blood pressure and preventing cardiovascular disease ${ }^{[65,66,67]}$ and are suitable for the control of any stage of blood pressure in the HBP 
population (including HBP patients and high-risk populations). Particularly, interventions such as a rational diet, weight control, smoking and alcohol consumption cessation, and stress reduction can be

adopted in the early stages for high-risk groups ${ }^{[33,68,69]}$. Considering the commonalities between NAFLD and HBP management, our study primarily analysed the correlation between risk factors and some dietary habits, aiming to provide a reference basis for health management based on dietary management to help physicians formulate individualised dietary interventions for individuals at high risk of HBP among NAFLD patients, and to raise patients' awareness of healthy dietary management through health education, adopt interactive follow-ups to timely access and provide feedback on the implementation of individualised dietary management and the real-time dynamics of key risk indicators.

\section{Conclusion}

This study used Lasso model based on 10-fold cross-validation and RF model to screen risk factor indicators (Age, SBP, BMI, ALT, UREA, UA, and NEUT) associated with NAFLD combined with HBP in the elderly population. Logistic regression models were used to obtain a simple, effective, and economical nomogram to predict the risk of comorbid HBP in NAFLD patients $\geq 60$ years in Shanghai, China.

Based on the results, the main management approach for elderly patients with NAFLD at risk for HBP lies in lifestyle changes, including a healthy and balanced diet, weight loss, and regular physical activity. Health service providers can formulate and improve individualised intervention measures and risk monitoring systems to increase patients' quality of life and reduce the economic and medical burden imposed by NAFLD and HBP on the elderly, their families, and society.

This study still has potential limitations. First, this is a retrospective study, and some non-conforming data were eliminated, which may lead to selection deviation. This risk-prediction model has been validated in the external validation population, but it lacks further validation in other populations in other regions and countries. Therefore, the model needs to be externally evaluated in a wider elderly population and needs further prospective research. This will confirm whether the individual intervention based on the prediction results of the model can reduce the risk of HBP in patients with NAFLD and improve living standards of NAFLD patients.

\section{Materials And Methods}

\subsection{Sample collection}

The sample data for this retrospective study were obtained from the Zhangjiang, Pudong New Area, Shanghai. We relied on the Shanghai Collaborative Innovation Center of Traditional Chinese Medicine Health Service of the Shanghai University of Traditional Chinese Medicine and the Zhangjiang and Beicai Community Health Service Centers, Pudong New Area, Shanghai, to collect the relevant information regarding the participants belonging to the Han population who participated in the chronic disease health screening programs conducted at the two community health centers from April 2016 to July 2017 using 
questionnaires, physical examinations, and biochemical tests. This study was conducted in accordance with the basic principles of the Declaration of Helsinki, and the protocol was approved by the Ethics Committee of the Shanghai University of Traditional Chinese Medicine. Informed consent was obtained from the study subjects, and they signed an informed consent form.

The research group collected 6,664 cases in 2017, who were matched for age and sex, randomised, and did not have blood relationship among the study subjects. After data processing, study subjects with missing information, incomplete indicators, and $<60$ years of age were excluded. The 5,287 cases with valid data were divided into two groups, a NAFLD group $(\mathrm{N}=2,845)$ and a non-NAFLD group $(\mathrm{N}=2,442)$. The NAFLD group was the internal training set, and the external validation set had 2,031 cases, which were obtained after preprocessing the data collected by the research group in 2016.

Blood pressure was measured using the steps recommended by international guidelines, including a quiet rest for at least 5 minutes before measurement. An electronic sphygmomanometer (Biospace, Cheonan, Korea) was used to record each subject's SBP and DBP ( $\mathrm{mmHg}$ ).

The clinical diagnostic criteria in the "Guidelines for the diagnosis and management of nonalcoholic fatty liver disease (2010 Revision)" developed by the Chinese Society of Hepatology, CMA ${ }^{[20]}$, was referred to to determine the diagnostic criteria for NAFLD. The following three conditions had to be met:

(1) No history of alcohol consumption or quantity of ethanol consumption $<140 \mathrm{~g} /$ week for men and $<70$ $\mathrm{g} /$ week for women.

(2) Exclusion of other causes that could lead to liver steatosis, including excessive alcohol consumption, viral hepatitis, drug-induced liver disease, autoimmune liver disease, hepatolenticular degeneration, and total parenteral nutrition.

(3) Imaging or histological findings should have met the diagnostic criteria of fatty liver disease.

\subsection{Statistical and modelling methods}

The analysis software used in this study were IBM SPSS Statistics (version: 25.0), Venny (version: 2.1.0), and R software (version: 4.1.0), and the R packages were caret, DynNom, haven, readxl, rms, rmda, regplot, rsconnect, glmnet, nricens, pROC, and forestplot. The statistical tests used were all two-sided tests with test level $a=0.05$.

The Lasso model based on 10-fold cross-validation and RF model were used to screen the risk factors of HBP in patients with NAFLD. The Lasso model is suitable for variable screening of high-dimensional data, where the variables in the analysis are first pooled and normalised to shrink the regression coefficients for the variables toward zero, and the predictor variables with non-zero coefficients at the best lambda value are screened with the smallest possible prediction error ${ }^{[21,22]}$. The RF model is based on a recursive feature elimination approach that uses significant variables in the hierarchical ranking to train the 
random forest and eliminate irrelevant variables, followed by the repeated training and elimination processes until there is no variable change ${ }^{[23]}$.

In this study, the outcome variable was whether HBP occurred under NAFLD conditions. As it is a dichotomous variable, logistic regression analysis was used to construct a prediction model containing the final characteristic variables ${ }^{[15]}$. The Lasso model was intersected with the predictor variables screened using RF, and the screened common variables were included in the multivariate logistic regression analysis. The $p>0.05$ predictor variables were gradually eliminated to obtain the final characteristic variables of the risk-prediction model, and the results were visualised using forest plots.

A nomogram can simplify and visualise complex regression equations, integrate data with a model, comprehensively consider the influential variables in the analysis, and graphically display the probability of the predicted outcome ${ }^{[24]}$. Therefore, we used the rms package in R software to plot the nomogram of the logistic regression model constructed in this study. The length of the line corresponding to each analysed variable in the plot indicated the degree of influence of that variable on the predicted outcome $\mathrm{e}^{[25]}$.

\subsection{Model evaluation and comparison}

We used the area under the ROC curve (AUC) to evaluate the discriminatory ability of the model in internal and external validation sets ${ }^{[26]}$. The value is generally between 0.5 and 1 , and the closer to 1 , the better the discriminatory ability of the model and the higher the predictive performance ${ }^{[18]}$. Appropriate calibration analysis is required when applying the prediction model constructed in this study to clinical decision-making ${ }^{[27]}$; therefore, we used the calibration curve to determine the degree of agreement between the actual status and the predicted results of the model. Because there is a possibility of false positives or false negatives in the prediction results of this model, we used DCA to determine the net benefit of the prediction model in clinical applications ${ }^{[15]}$. The NRI proposed in 2008 to evaluate the improvement of a new model in risk-prediction ability ${ }^{[28]}$ was applied in this study to compare the extent to which the newly constructed prediction model outperformed the prediction model constructed based on the traditional variables used in HBP diagnosis.

The flow chart of this study design is shown in Figure 1.

\section{Declarations}

\section{Data availability}

The datasets generated and analysed during the current study are available from the corresponding author on reasonable request.

\section{Acknowledgements}


The authors thank all the participating community health centers that helped recruit the participants, and all participants who volunteered to be investigated. We would like to thank Editage (www.editage.cn) for English language editing.

\section{Funding}

This study was supported by grants from the Tracking and Evaluation project of Implementation Effect of National Basic Public Health Service Project in Shanghai of Shanghai Municipal Health Commission (No. HWJC201901).

\section{Author contributions}

J.J.S. and A.Z. conceived and designed the research; B.C.L and D.L. collected the data; X.L. and X.X.S. sorted the data; X.L. processed the statistical analysis and composed the first draft of the manuscript; J.J.S. and A.Z. checked and corrected the draft. All authors read and approved the final manuscript.

These authors contributed equally: X.L. and A.Z.

\section{Competing interest statement}

The authors declare no competing interests.

\section{References}

1. Vancells Lujan, P., Viñas Esmel, E. \& Sacanella Meseguer, E. Overview of non-alcoholic fatty liver disease (Nafld) and the role of sugary food consumption and other dietary components in its development. Nutrients 13, (2021).

2. Younossi, Z. M. et al. Global epidemiology of nonalcoholic fatty liver disease-Meta-analytic assessment of prevalence, incidence, and outcomes. Hepatology 64, 73-84 (2016).

3. Francque, S. M. et al. Non-alcoholic fatty liver disease: A patient guideline. JHEP Reports 3, 100322 (2021).

4. Shegaze, M., Adal, T., Mesfin, D. \& Shibiru, T. The burden of cancer in Ethiopia, 2000-2016: analysis of evidence from the Global Burden of Disease study and Global Health Estimate. Med. Stud. 36, 8389 (2020).

5. Mahady, S. E. \& George, J. Predicting the future burden of NAFLD and NASH. J. Hepatol. 69, 774775 (2018).

6. Zhou, B., Perel, P., Mensah, G. A. \& Ezzati, M. Global epidemiology, health burden and effective interventions for elevated blood pressure and hypertension. Nat. Rev. Cardiol. 18, 785-802 (2021).

7. Cai, X. et al. Development and Validation of a Novel Model for Predicting the 5-Year Risk of Type 2 Diabetes in Patients with Hypertension: A Retrospective Cohort Study. Biomed Res. Int. 2020, (2020). 
8. Gakidou, E. et al. Global, regional, and national comparative risk assessment of 84 behavioural, environmental and occupational, and metabolic risks or clusters of risks, 1990-2016: A systematic analysis for the Global Burden of Disease Study 2016. Lancet 390, 1345-1422 (2017).

9. Chinese Nutrition Society. Report on the State of Nutrition and Chronic Diseases in China (2021). (2021).

10. López-Suárez, A. et al. Nonalcoholic fatty liver disease is associated with blood pressure in hypertensive and nonhypertensive individuals from the general population with normal levels of alanine aminotransferase. Eur. J. Gastroenterol. Hepatol. 23, 1011-1017 (2011).

11. Feng, R. N. et al. Lean-non-alcoholic fatty liver disease increases risk for metabolic disorders in a normal weight Chinese population. World J. Gastroenterol. 20, 17932-17940 (2014).

12. Campos-Murguía, A., Ruiz-Margáin, A., González-Regueiro, J. A. \& Macías-Rodríguez, R. U. Clinical assessment and management of liver fibrosis in nonalcoholic fatty liver disease. World $\mathrm{J}$. Gastroenterol. 26, 5919-5943 (2020).

13. Zhao, Y. C. et al. Nonalcoholic Fatty Liver Disease: An Emerging Driver of Hypertension. Hypertension 275-284 (2020) doi:10.1161/HYPERTENSIONAHA.119.13419.

14. Jichitu, A. et al. Non-alcoholic fatty liver disease and cardiovascular comorbidities: Pathophysiological links, diagnosis, and therapeutic management. Diagnostics 11, (2021).

15. Zhou, Z.-R. et al. In-depth mining of clinical data: the construction of clinical prediction model with R. Ann. Transl. Med. 7, 796-796 (2019).

16. Sun, D. et al. Recent development of risk-prediction models for incident hypertension: An updated systematic review. PLoS One 12, 1-19 (2017).

17. Ren, Z. et al. A novel predicted model for hypertension based on a large cross-sectional study. Sci. Rep. 10, 1-9 (2020).

18. Xue, M. et al. A simple nomogram score for screening patients with type 2 diabetes to detect those with hypertension: A cross-sectional study based on a large community survey in China. PLoS One $15,1-15$ (2020).

19. Deng, X. et al. Development and validation of a nomogram to better predict hypertension based on a 10-year retrospective cohort study in China. Elife 10,1-19 (2021).

20. Fan, J. G. et al. Guidelines for the diagnosis and management of nonalcoholic fatty liver disease: Update 2010: (Published in Chinese on Chinese Journal of Hepatology 2010; 18:163-166) JG Fan etal. Diagnosis and management of NAFLD. J. Dig. Dis. 12, 38-44 (2011).

21. Zhang, Y. et al. Establishment of a Risk Prediction Model for Non-alcoholic Fatty Liver Disease in Type 2 Diabetes. Diabetes Ther. 11, 2057-2073 (2020).

22. Wang, H. et al. Predicting medication nonadherence risk in a Chinese inflammatory rheumatic disease population: development and assessment of a new predictive nomogram. Patient Prefer. Adherence 12, 1757-1765 (2018). 
23. Le Thi, H. A., Nguyen, V. V. \& Ouchani, S. Gene selection for cancer classification using DCA. Lect. Notes Comput. Sci. (including Subser. Lect. Notes Artif. Intell. Lect. Notes Bioinformatics) 5139 LNAl, 62-72 (2008).

24. Liu, Q. et al. Development and validation of prognostic nomogram for lung cancer patients below the age of 45 years. Bosn. J. Basic Med. Sci. 21, 620-631 (2021).

25. Xu, R. et al. Development and validation of prognostic nomograms for patients with colon neuroendocrine neoplasms. World J. Surg. Oncol. 19, 477-492 (2021).

26. Unal, I. Defining an optimal cut-point value in ROC analysis: An alternative approach. Comput. Math. Methods Med. 2017, (2017).

27. Huang, Y., Li, W., Macheret, F., Gabriel, R. A. \& Ohno-Machado, L. A tutorial on calibration measurements and calibration models for clinical prediction models. J. Am. Med. Informatics Assoc. 27, 621-633 (2021).

28. Pencina, M. J., D’Agostino Sr, R. B., D’Agostino Jr, R. B. \& Vasan, R. S. Evaluating the added predictive ability of a new marker: From area under the ROC curve to reclassification and beyond. Stat. Med. 27, 157-172 (2008).

29. Zhang, W. et al. Identification of hypertension predictors and application to hypertension prediction in an urban Han Chinese population: A longitudinal study, 2005-2010. Prev. Chronic Dis. 12, 1-10 (2015).

30. Chen, Y. et al. Incident hypertension and its prediction model in a prospective northern urban Han Chinese cohort study. J. Hum. Hypertens. 30, 794-800 (2016).

31. Harvey, A., Montezano, A. C. \& Touyz, R. M. Vascular biology of ageing-Implications in hypertension. J. Mol. Cell. Cardiol. 83, 112-121 (2015).

32. Wang, M., Monticone, R. E. \& Lakatta, E. G. Arterial aging: A journey into subclinical arterial disease. Curr. Opin. Nephrol. Hypertens. 19, 201-207 (2010).

33. Liu, L. S. et al. 2018 Chinese guidelines for prevention and treatment of hypertension - A report of the revision committee of Chinese guidelines for prevention and treatment of hypertension. J. Geriatr. Cardiol. 16, 182-245 (2019).

34. Feng, R. N. et al. BMI is strongly associated with hypertension, and waist circumference is strongly associated with type 2 diabetes and dyslipidemia, in Northern Chinese adults. J. Epidemiol. 22, 317323 (2012).

35. Zhou, B. F. Predictive values of body mass index and waist circumference for risk factors of certain related diseases in Chinese adults--study on optimal cut-off points of body mass index and waist circumference in Chinese adults. Biomed. Environ. Sci. 15, 83-96 (2002).

36. Egan, B. M., Li, J., Hutchison, F. N. \& Ferdinand, K. C. Hypertension in the United States, 1999 to 2012: progress toward Healthy People 2020 goals. Circulation 130, 1692-1699 (2014).

37. Younossi, Z. et al. Global burden of NAFLD and NASH: trends, predictions, risk factors and prevention. Nat. Rev. Gastroenterol. Hepatol. 15, 11-20 (2018). 
38. Gildner, T. E., Barrett, T. M., Liebert, M. A., Kowal, P. \& Snodgrass, J. J. Does BMI generated by selfreported height and weight measure up in older adults from middle-income countries? Results from the study on global AGEing and adult health (SAGE). BMC Obes. 2, 1-13 (2015).

39. Bulpitt, C. J. \& Breckenridge, A. Plasma urea in hypertensive patients. Heart 38, 689-694 (1976).

40. Zhang, Z., Dmitrieva, N. I., Park, J. H., Levine, R. L. \& Burg, M. B. High urea and NaCl carbonylate in renal cells in culture and in vivo, and high urea causes 8-oxoguanine lesions in their DNA. Proc. Natl. Acad. Sci. U. S. A. 101, 9491-9496 (2004).

41. Weiner, I. D., Mitch, W. E. \& Sands, J. M. Urea and ammonia metabolism and the control of renal nitrogen excretion. Clin. J. Am. Soc. Nephrol. 10, 1444-1458 (2015).

42. Polychronopoulou, E., Wuerzner, G. \& Burnier, M. How do I manage hypertension in patients with advanced chronic kidney disease not on dialysis? Perspectives from clinical practice. Vasc. Health Risk Manag. 17, 1-11 (2021).

43. Roumeliotis, S., Roumeliotis, A., Dounousi, E., Eleftheriadis, T. \& Liakopoulos, V. Dietary antioxidant supplements and uric acid in chronic kidney disease: A review. Nutrients 11, 1-18 (2019).

44. Hirai, A. \& Saitoh, Y. [Hypertension and hyperuricemia]. Nihon Rinsho. 54, 3283-3288 (1996).

45. Chen, H. et al. White blood cell count: an independent predictor of coronary heart disease risk in middle-aged and elderly population with hyperuricemia. Med. (United States) 97, 1-5 (2018).

46. Lombardi, R., Pisano, G. \& Fargion, S. Role of serum uric acid and ferritin in the development and progression of NAFLD. Int. J. Mol. Sci. 17, (2016).

47. Haim, M., Boyko, V., Goldbourt, U., Battler, A. \& Behar, S. Predictive Value of Elevated White Blood Cell Count in Patients With Preexisting Coronary Heart Disease: The Bezafibrate Infarction Prevention Study. Arch. Intern. Med. 164, 433-439 (2004).

48. Tatsukawa, Y. et al. White blood cell count, especially neutrophil count, as a predictor of hypertension in a Japanese population. Hypertens. Res. 31, 1391-1397 (2008).

49. McCarthy, C. et al. Innate Immune Cells and Hypertension: Neutrophils and Neutrophil Extracellular Traps (NETs). Compr. Physiol. 11, 1575-1589 (2021).

50. Aydın, M. Neutrophil lymphocyte ratio in obese adolescents. North. Clin. Istanbul 2, 87-91 (2015).

51. Brotfain, E. et al. Neutrophil functions in morbidly obese subjects. Clin. Exp. Immunol. 181, 156-163 (2015).

52. Liu, H. et al. The association between AST/ALT ratio and all-cause and cardiovascular mortality in patients with hypertension. Medicine (Baltimore). 100, e26693 (2021).

53. JM, M. et al. Aminotransferase Levels and 20-year Risk of Metabolic Syndrome, Diabetes, and Cardiovascular Disease. Bone 23, 1-7 (2008).

54. Wu, L. et al. Gender difference in the association between aminotransferase levels and hypertension in a Chinese elderly population. Med. (United States) 96, 1-7 (2017).

55. Jia, J. et al. The association between serum alanine aminotransferase and hypertension: A national based cross-sectional analysis among over 21 million Chinese adults. BMC Cardiovasc. Disord. 21, 
$1-12(2021)$.

56. Rose, G. et al. Intersalt: An international study of electrolyte excretion and blood pressure. Results for 24 hour urinary sodium and potassium excretion. Br. Med. J. 297, 319-328 (1988).

57. Sakaguchi, K., Takemi, Y., Hayashi, F., Koiwai, K. \& Nakamura, M. Effect of workplace dietary intervention on salt intake and sodium-to-potassium ratio of Japanese employees: A quasiexperimental study. J. Occup. Health 63, 1-12 (2021).

58. Huang, L. et al. Effect of dose and duration of reduction in dietary sodium on blood pressure levels: Systematic review and meta-analysis of randomised trials. BMJ 368, 8-10 (2020).

59. Hipgrave, D. B., Chang, S., Li, X. \& Wu, Y. Salt and sodium intake in China. JAMA - J. Am. Med. Assoc. 315, 703-705 (2016).

60. Bröer, S. \& Bröer, A. Amino acid homeostasis and signalling in mammalian cells and organisms. Biochem. J. 474, 1935-1963 (2017).

61. Hollenberg, N. K. Effects on blood pressure of reduced dietary sodium and the dietary approaches to stop hypertension (DASH) diet: Editor's comments. Curr. Hypertens. Rep. 3, 373 (2001).

62. Filippou, C. D. et al. Dietary Approaches to Stop Hypertension (DASH) Diet and Blood Pressure Reduction in Adults with and without Hypertension: A Systematic Review andMeta-Analysis of Randomized Controlled Trials Christina. 1150-1160 (2020).

63. Moore, M. P., Cunningham, R. P., Dashek, R. J., Mucinski, J. M. \& Rector, R. S. A Fad too Far? Dietary Strategies for the Prevention and Treatment of NAFLD. Obesity 28, 1843-1852 (2020).

64. Stachowska, E. et al. Nutritional strategies for the individualized treatment of non-alcoholic fatty liver disease (NAFLD) based on the nutrient-induced insulin output ratio (NIOR). Int. J. Mol. Sci. 17, (2016).

65. Appel, L. et al. Dietary Approaches to Prevent and Treat Hypertension A Scientific Statement From the American Heart Association. Hypertension 47, 296-308 (2006).

66. Appel, L. J. Lifestyle modification as a means to prevent and treat high blood pressure. J. Am. Soc. Nephrol. 14, 99-102 (2003).

67. Carey, R. M., Muntner, P., Bosworth, H. B. \& Whelton, P. K. Reprint of: Prevention and Control of Hypertension: JACC Health Promotion Series. J. Am. Coll. Cardiol. 72, 2996-3011 (2018).

68. Aburto, N. J. et al. Effect of increased potassium intake on cardiovascular risk factors and disease: Systematic review and meta-analyses. BMJ 346, 1-19 (2013).

69. Landsbergis, P. A., Dobson, M., Koutsouras, G. \& Schnall, P. Job strain and ambulatory blood pressure: A meta-analysis and systematic review. Am. J. Public Health 103, 61-71 (2013).

\section{Figures}




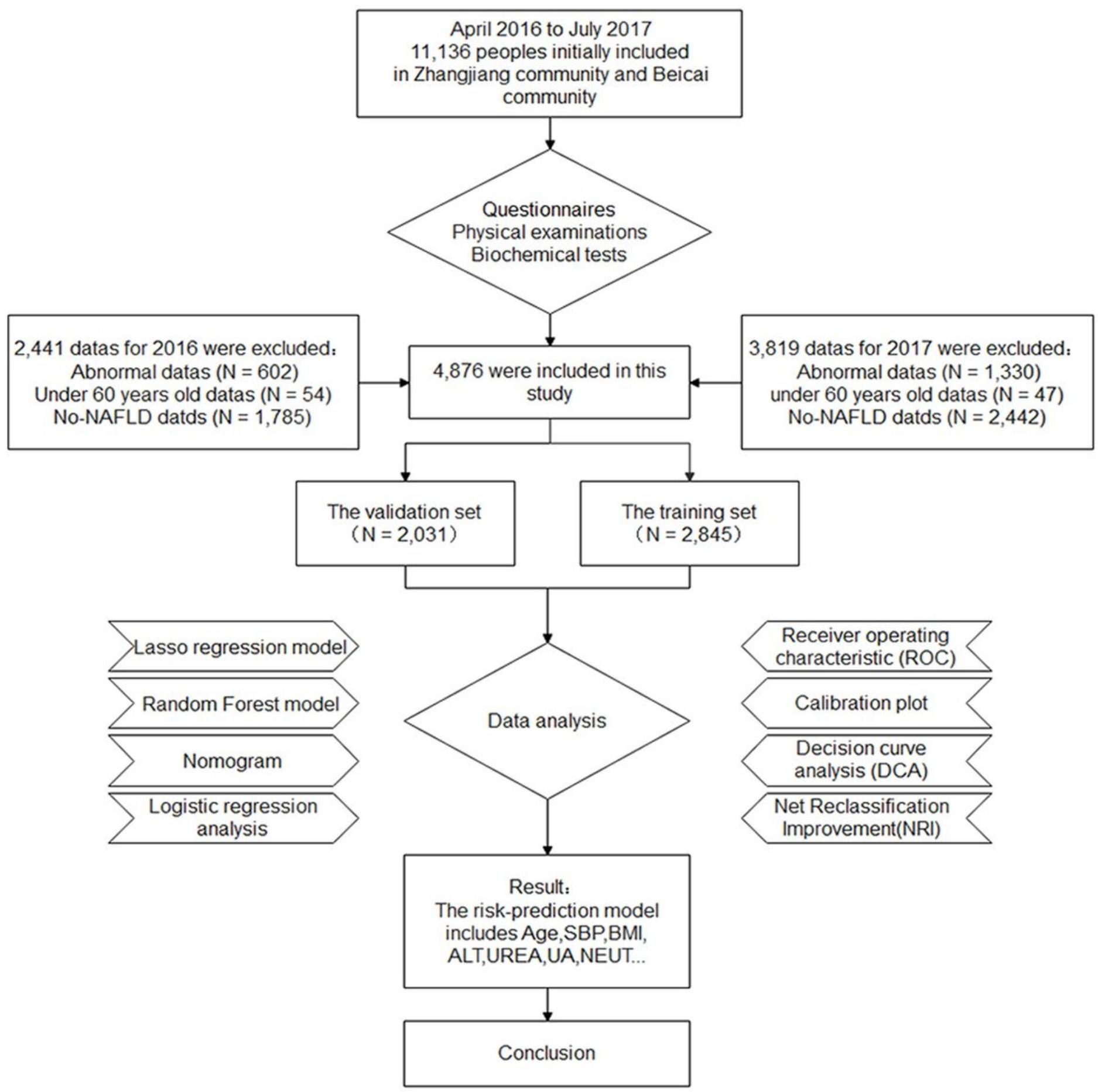

\section{Figure 1}

Flow diagram of the study design 
A

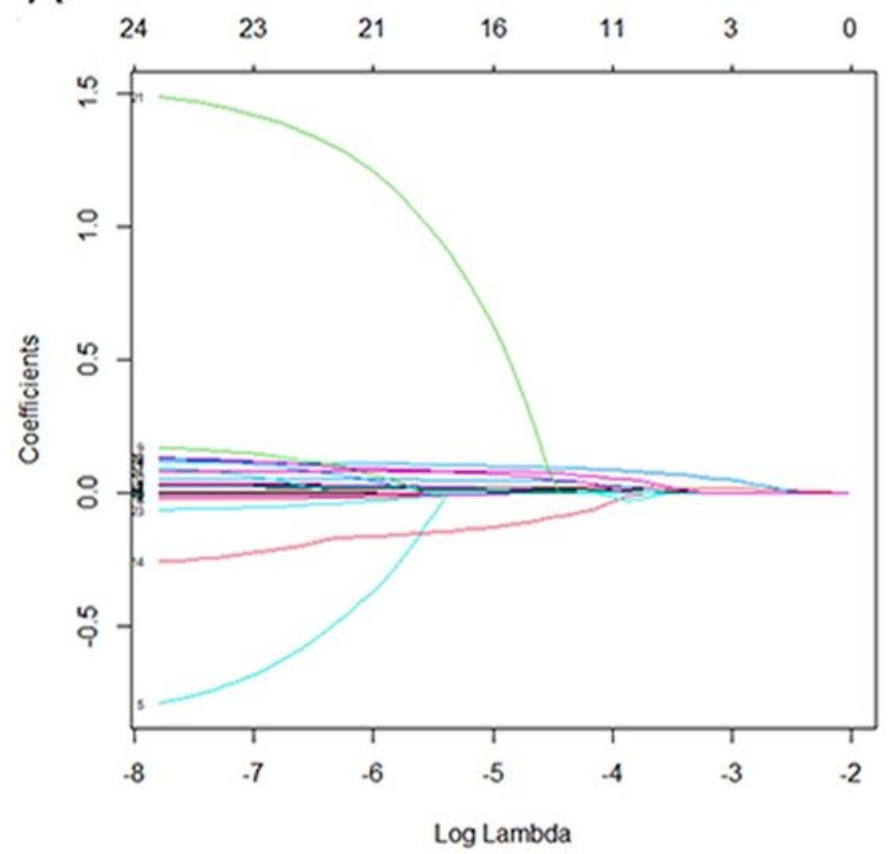

$\mathrm{C}$

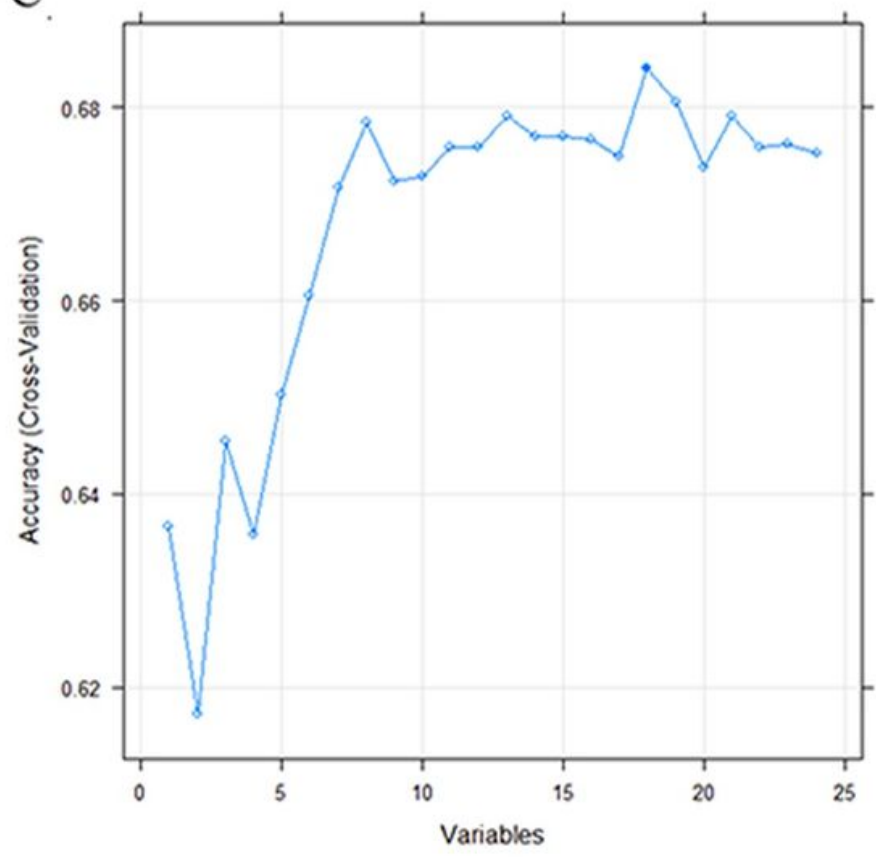

B

$\begin{array}{llllllllllllll}24 & 24 & 23 & 23 & 21 & 19 & 16 & 14 & 11 & 9 & 5 & 3 & 2 & 1\end{array}$

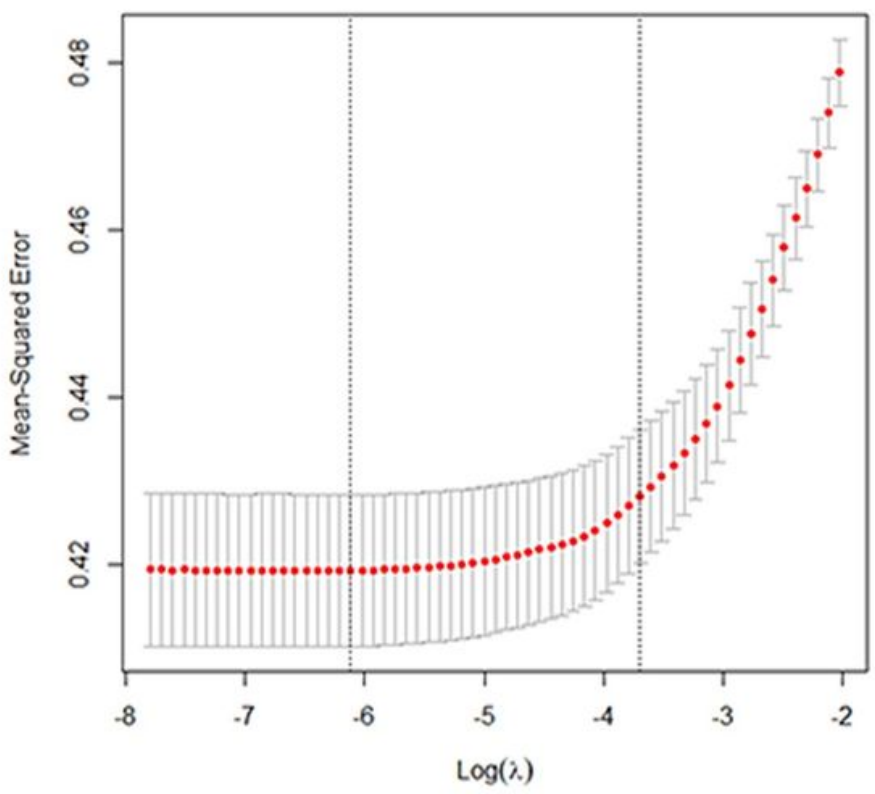

$\mathrm{D}$

\section{Lasso Regression Random Forest}

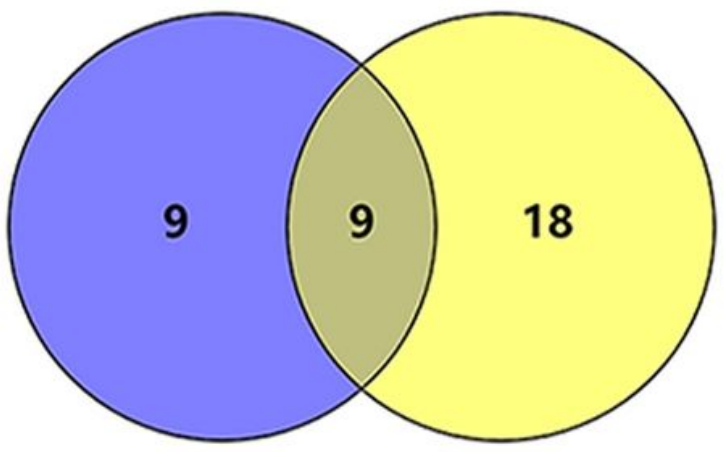

Figure 2

Variable screening using the Lasso regression model. (A) Nine variables with non-zero coefficients were selected by deriving the optimal lambda values. (B) Coefficient profiles are generated according to optimal parameters (lambda) in Figure 2(A). (C) Result graph of the variable screened by the random forest model method. (D) Schematic diagram of the method for screening the final characteristic variables in this study. 
Variables

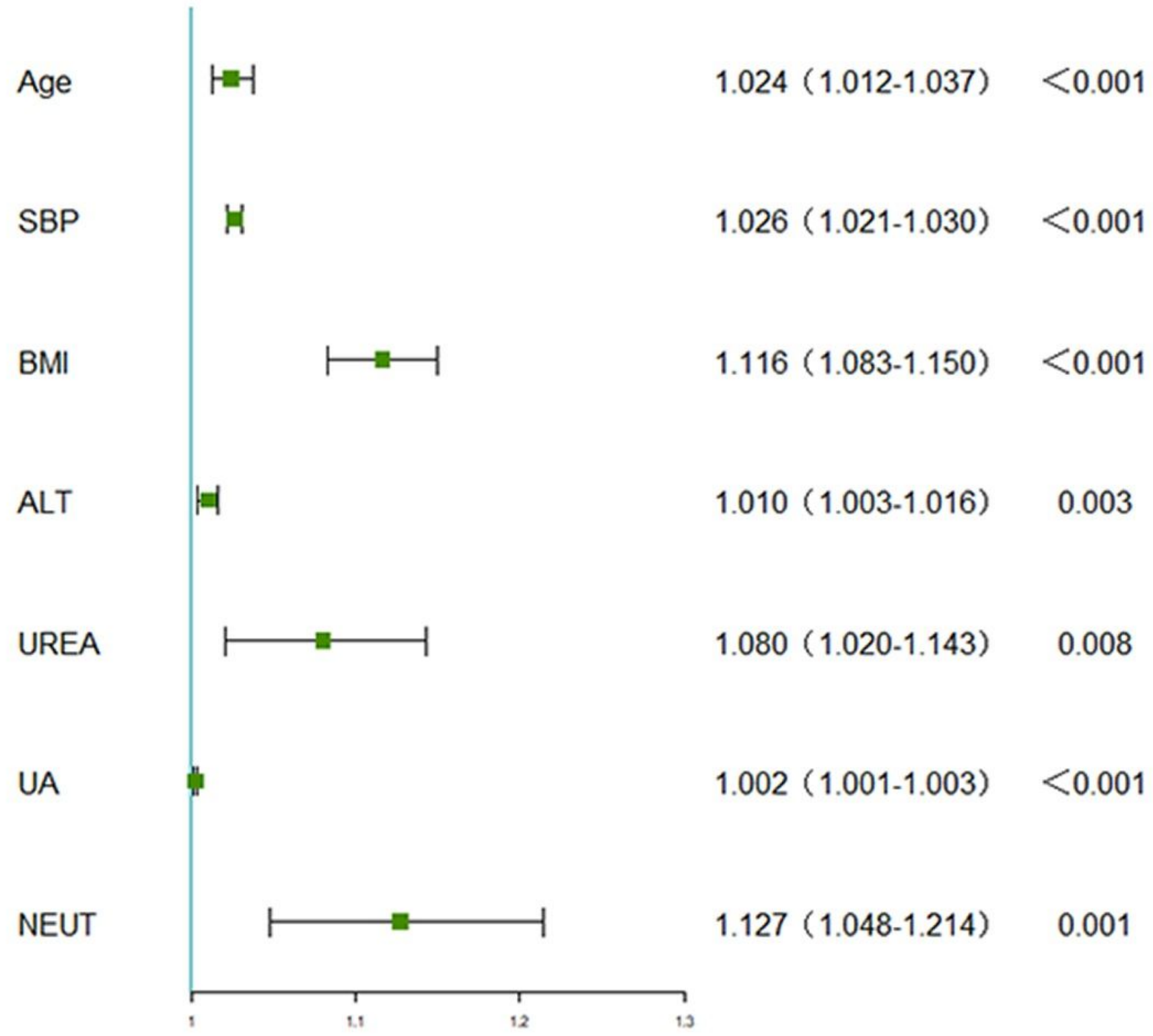

Figure 3

Forest plot of selected elements. Forest plot is used to visualise the results of logistic regression analysis. 
A

Points

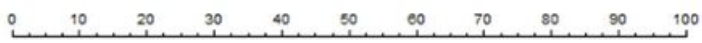

Age

${ }_{00}^{1} 70 \quad 8090 \quad 105$

SBP

BMI

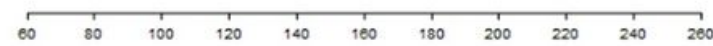

ALT

UREA

UA

$\begin{array}{llllll}100 & 250 & 400 & 580 & 700\end{array}$

NEUT

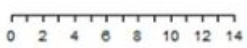

Total Points

Risk of Hypertension
B

Points

UREA ${ }^{\star \star x}$

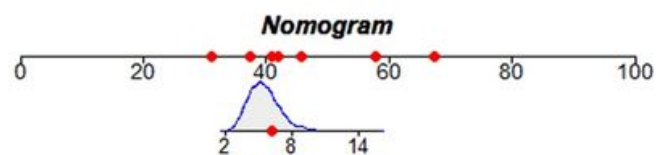

ALT**

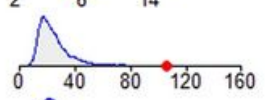

NEUT ${ }^{\star \star}$

$\mathrm{Age}^{\star \star \star x}$

$U A^{\star \star \star}$

$B \mathrm{M}^{* \star *}$

$\mathrm{SBP}^{\star x \times x}$

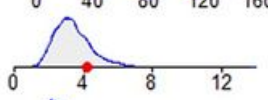

Total points

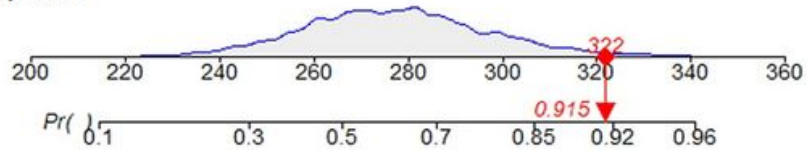

Figure 4

The risk nomogram of HBP in patients with NAFLD. (A) Nomogram including Age, SBP, BMI, ALT, UREA, TC, LDL, UA, and NEUT for analysis. (B) One patient with NAFLD was randomly selected from the sample, which's HBP incidence was predicted based on the seven characteristic indicators of the nomogram.
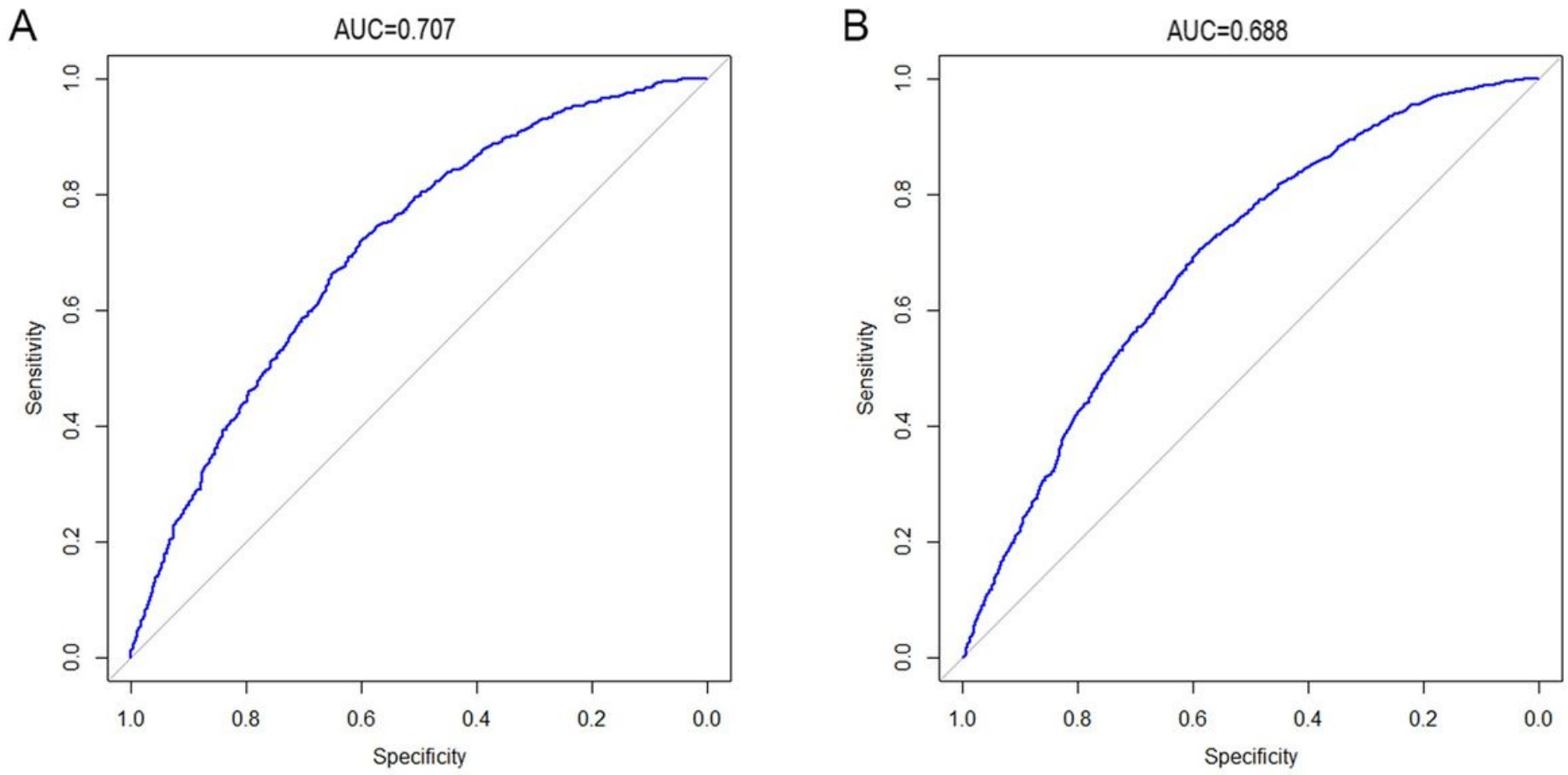

Figure 5 
The area under the ROC curve is used to determine the predictive ability of the prediction model to distinguish outcomes, such as with or without disease. X-axis represents the false positive rate predicted by the model; Y-axis represents the true positive rate predicted by the model; blue curve represents the performance of the nomogram. (A) Performance of the model in the training set. (B) Performance of the model in the validation set.

A

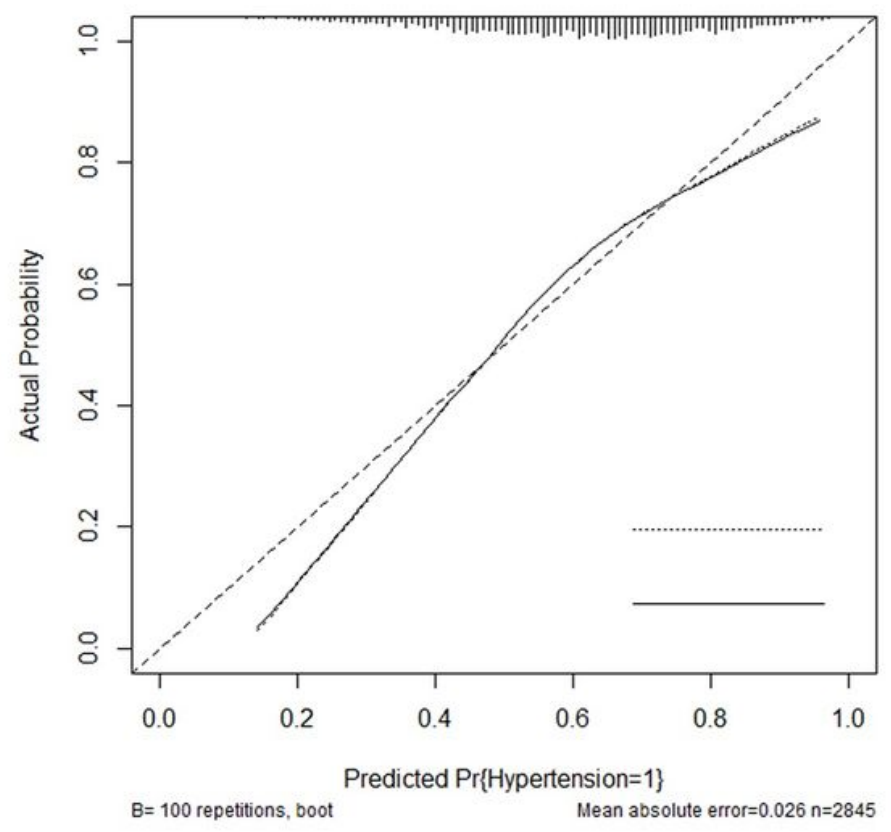

B

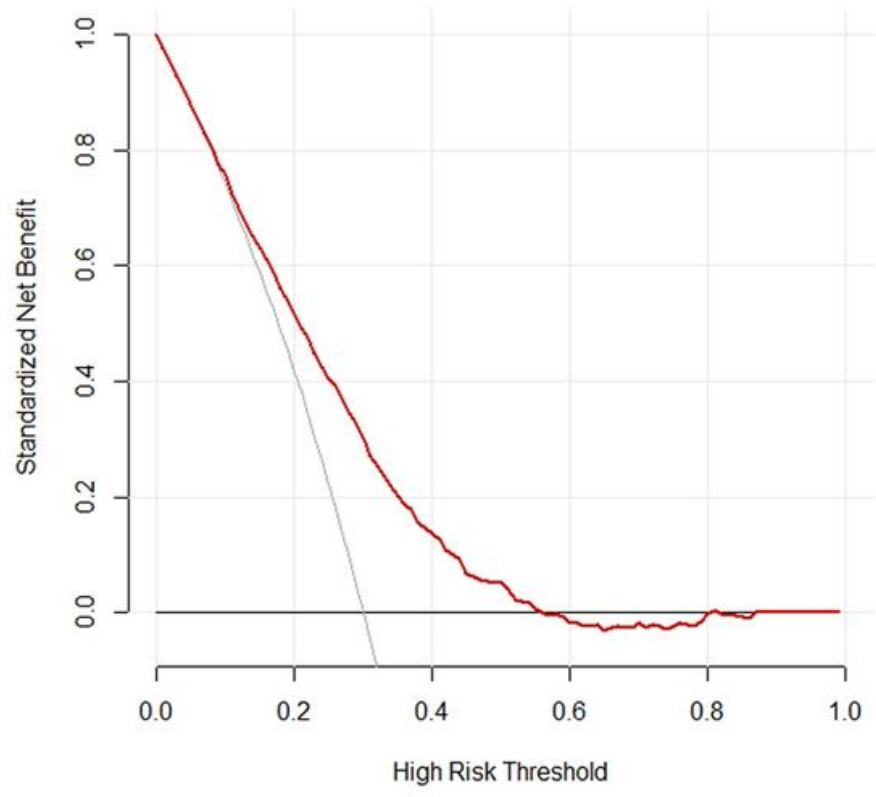

\section{Figure 6}

(A) X-axis of the curve represents the predicted risk of concurrent HBP in elderly patients with NAFLD, Yaxis represents the actual diagnosed morbidity, the diagonal line represents the perfect prediction of the ideal model, and the higher overlap of the dark dashed line with the diagonal line represents the better accuracy of the model. (B) Decision curve analysis of the HBP risk nomogram. Solid line parallel to the horizontal axis in the graph indicates that when all samples are negative, the net benefit of having no intervention for all is 0 . Backlash represents that all samples are positive and have received intervention, and the net benefit is the magnitude of the slope. Red line represents the net benefit of the model constructed in this study. The further the red line is from the two intersecting lines, the higher the clinical application value of the model. 


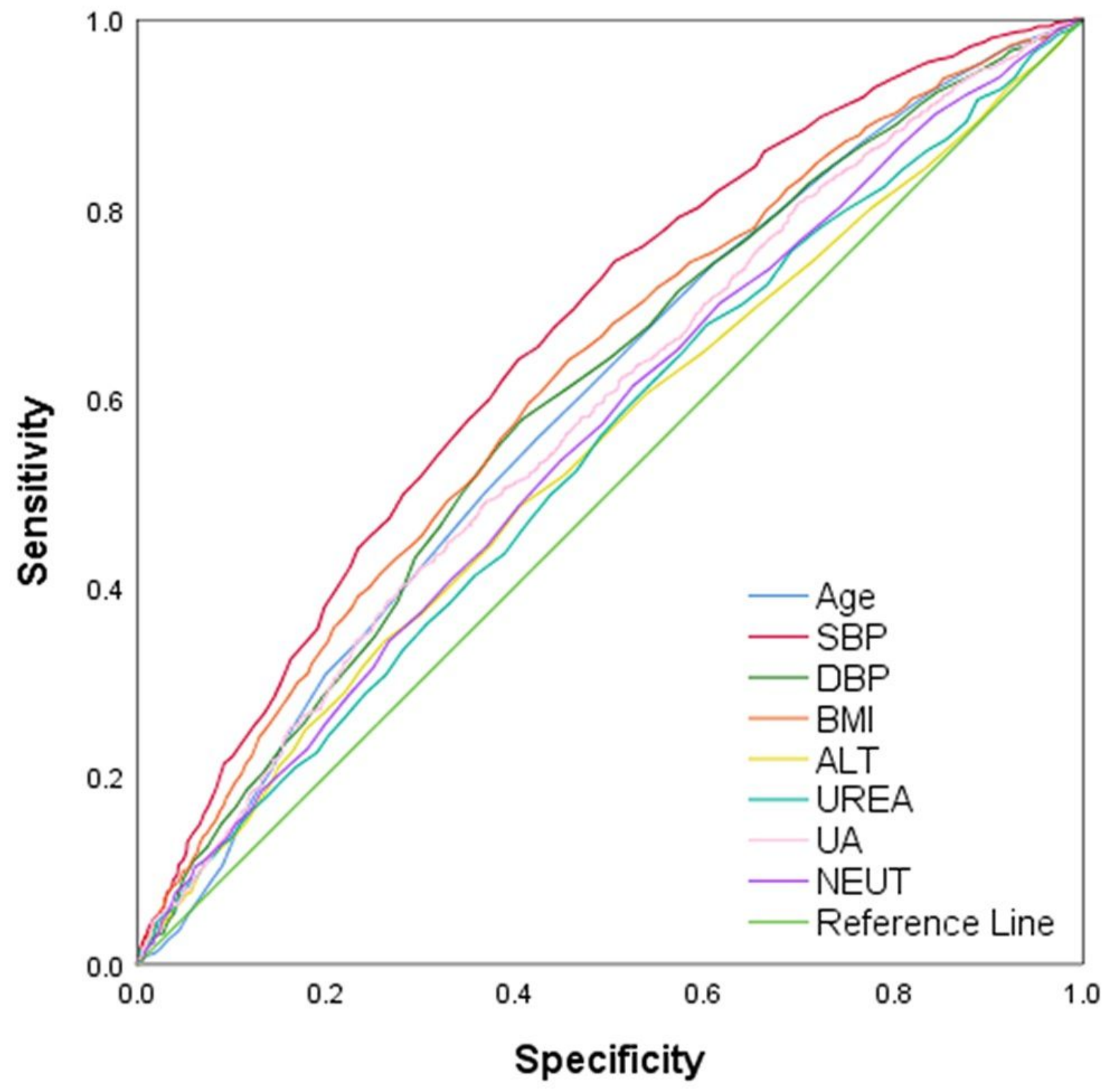

Figure 7

ROC curve analysis of the seven risk factors. 


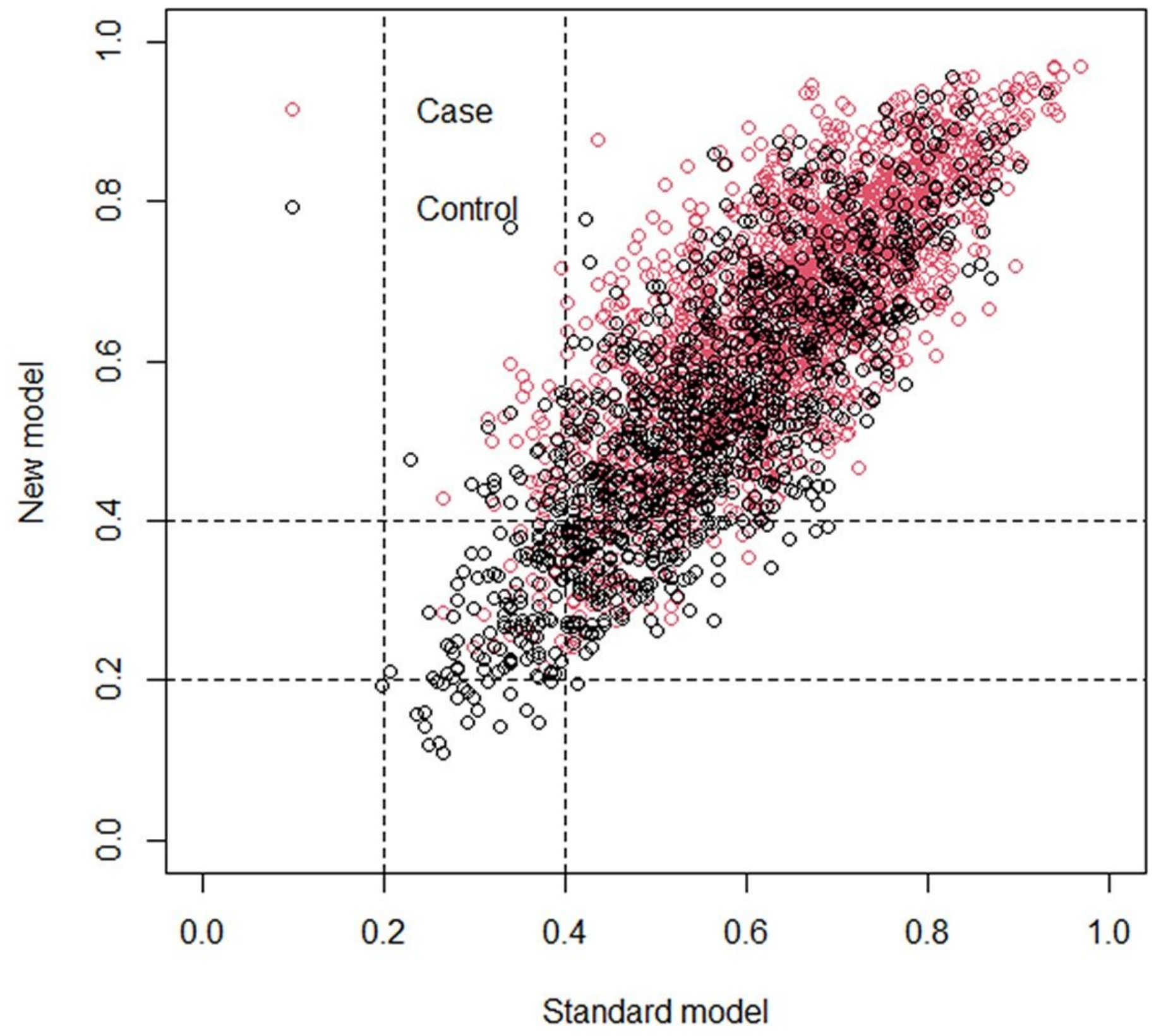

Figure 8

Model comparison based on NRI. The NRI was 0.109. 